\title{
Design and evaluation of a multi-epitope assembly Peptide (MEAP) against herpes simplex virus type 2 infection in BALB/c mice
}

Xingsheng Wang ${ }^{1 \dagger}$, Guangyan Xie ${ }^{3 \dagger}$, Jianming Liao ${ }^{2}$, Dengke Yin ${ }^{3}$, Wenyan Guan ${ }^{1}$, Mingjie Pan ${ }^{1}$, Jingnian $\mathrm{Li}^{4}$ and Yuexi Li $i^{* *}$

\begin{abstract}
Background: Human herpes simplex virus (HSV) 1 and 2 causes oral, ocular, or genital infections, which remains a significant health problem worldwide. HSV-1 and -2 infections in humans range from localized skin infections of the oral, ocular, and genital regions to severe and often disseminated infections in immunocompromised hosts. Epitope based vaccination is a promising mean to achieve protective immunity and to avoid infections with Human herpes simplex virus type 2 (HSV-2).

Methods: The twelve selected epitopes, six B cell epitopes from different glycoprotein of HSV-2 (amino acid residues 466-473 (EQDRKPRN) from envelope glycoprotein B, 216-223 (GRTDRPSA) from C, 6-18 (DPSLKMADPNRFR) from D, 483-491 (DPPERPDSP) from E, 572-579 (EPPDDDDS) from $G$ and 286-295 (CRRRYRRPRG) from I glycoprotein of HSV-2), four CD4 ${ }^{+} T$ cell epitopes (amino acid residues 21-28 (NLPVLDQL) from D, 162-177

(KDVTVSQWWFGHRYSQ) from B, 205-224 (KAYQQGVTVDSIGMLPRFIP) from D and 245-259 (KPPYTSTLLPPELSD) from D) and two CD8 ${ }^{+} \mathrm{T}$ cell epitopes (amino acid residues 10-20 (KMADPNRFRGK) from D and 268-276 (ALLEDPAGT) from D), are responsible for the elicitation of the neutralizing antibodies and cytotoxic $T$ lymphocytes (CTLs) that impart protective immunity to the host. In this study, all above epitopes were inserted into the extracellular fragment (amino acid residues 1-290) of HSV-2 glycoprotein D to construct multi-epitope assembly peptides (MEAPs) by replacing some non-epitope amino acid sequences. The epitope independency of the MEAPs was predicted by three-dimensional software algorithms. The gene of the selected MEAP was expressed in E.coli BL21 (DE3), and its protective efficacy against HSV-2 infection was assessed in BALB/C mice.

Results: The MEAP, with each inserted epitopes independently displayed on the molecule surface, was selected as candidate proteins. The results showed that the MEAP was highly immunogenic and could elicit high titer neutralizing antibodies and cell-mediated immune responses.
\end{abstract}

Conclusions: The MEAP provided complete protection against infection with HSV-2 in mice, which indicates that it might be a potential candidate vaccine against HSV-2.

\section{Background}

Human herpes simplex virus (HSV) 1 and 2 cause oral, ocular, and genital infections, which construct a significant health problem worldwide. HSV-1 and -2 infections in humans range from localized skin infections of the oral, ocular, and genital regions to severe and often

\footnotetext{
* Correspondence: liyxi2007@126.com

+ Contributed equally

'Huadong Research Institute for Medicine and Biotechniques, Nanjing, 210002, China

Full list of author information is available at the end of the article
}

disseminated infections in immunocompromised hosts [1]. After primary infection of mucosal epithelial cells, the virus establishes lifelong latency in sensory neurons, from which it periodically reactivates [2]. After reactivation, the virus migrates along the axons and infects cells to the site of primary infection, causing painful blisters on the surface of the lips in the case of HSV-1, or of the genital mucosa for the closely related HSV-2 [3].

Four glycoproteins of the HSV-2, glycoprotein B (gB), $\mathrm{gD}, \mathrm{gH}$ and $\mathrm{gL}$, have essential functions for HSV-2

\section{Biomed Central}


entering into the host cells [4]. The cooperation of $\mathrm{gB}$, the heterodimer $\mathrm{gH} / \mathrm{gL}$, as well as $\mathrm{gD}$ and the $\mathrm{gD}$ receptor are required in entering the plasma or endosomal membrane of host cells [5,6]. The function of gD in viral infectivity has been associated with the adsorptionpenetration process. It binds to the host cell at the positions of 52, 60 and 197-199 of gD in the amino acid sequence. GB and gL, with the help from gK, are also importantly associated with the adsorption-penetration process $[7,8]$.

During the last decade, HSV vaccine development has primarily focused on various forms of recombinant glycoprotein. Recently, many approaches in vaccine development have appeared, including one chemically synthesized peptides covering only a small region of the amino acid sequence of a protein [9]. It was reported that B cell epitopes from the amino acid sequence of gD2 could induce mice to produce antibodies against a potent and type-common antiviral activity, and some B cell epitopes of HSV-2 glycoprotein have been identified [10].

Neutralization antibodies to HSV-2 (B cellular immunity) play a prominent role in prophylactic protection from infection in animal models, while $\mathrm{CD} 4^{+} \mathrm{T}$ cellbased cellular immunity to HSV-2 may play an important role in controlling recurrent human disease. Despite previous emphasis on antibody $(\mathrm{Ab})$ and $\mathrm{CD} 8^{+} \mathrm{T}$ cell responses, there is growing evidence to support a pivotal role for the $\mathrm{CD}_{4}{ }^{+} \mathrm{T}$ cells in antiherpesvirus immunity. $\mathrm{CD} 4^{+} \mathrm{T}$ cells are required for the protection of mice from HSV-2 challenge [11]. Severe herpetic infections are often seen in immunocompromised individuals with impaired $\mathrm{CD} 4^{+} \mathrm{T}$ cell immunity, such as those with AIDS and transplant patients, which indicate that $\mathrm{CD} 4^{+}$ $\mathrm{T}$ cells are very important for protection against virus infection. It is believed that $\mathrm{CD} 4^{+} \mathrm{T}$ cell responses are important for protection against HSV-2 infection. These findings, along with the important role of $\mathrm{CD} 4^{+} \mathrm{T}, \mathrm{CD} 8$ ${ }^{+} \mathrm{T}$ and $\mathrm{B}$ cells, suggested that a successful immunoprophylactic and immunotherapeutic strategy against HSV2 should include immunodominant $\mathrm{CD} 4^{+} \mathrm{T}$ and $\mathrm{CD} 8^{+}$ $\mathrm{T}$ cell epitopes.

It is necessary to develop an effective vaccine against HSV-2 that can stimulate the body to produce both cell-mediated and humoral immunity. Therefore, it is expected that a novel HSV-2 vaccine should be developed through the selection of immunodominant epitopes of $\mathrm{CD}^{+} \mathrm{T}, \mathrm{CD} 8^{+} \mathrm{T}$ and $\mathrm{B}$ cell. In this study, twelve selected epitopes, six B cell epitopes, four $\mathrm{CD} 4^{+}$ $\mathrm{T}$ and two $\mathrm{CD} 8^{+} \mathrm{T}$ cell epitopes, were all inserted into the extracellular fragment (1-290) of HSV-2 glycoprotein $D$ to construct multi-epitope assembly peptides (MEAPs) by replacing some non-epitope amino acid sequences. The results showed that the MEAP expressed by bioengineering had strong and specific antigenicity and was able to strongly induce Th1 and Th2 cytokine (IL-2, IL-4 and IFN- $\gamma$ ) secretion, providing a basis for the development of novel vaccines against HSV-2.

\section{Materials and methods \\ Virus and cell lines}

Vero cells were grown in medium 199 supplemented with $10 \%$ fetal calf serums $100 \mathrm{U} / \mathrm{ml}$ penicillin, $0.1 \mathrm{~g} / \mathrm{L}$ streptomycin (all from GIBCO, USA) for use. Target cells (NIH 3T3 cells) was kindly provided by Prof. Liu (Anhui Medical University, China) and used as target cells for CTL assay. Monolayers were infected with HSV-2, Sav strain. Inactivated virus was prepared by infection of cells at a multiplicity of 0.1 . After 4 days, extracellular virus was isolated and heat-inactivated for $30 \mathrm{~min}$ at $56^{\circ} \mathrm{C}$, and stored at $-80^{\circ} \mathrm{C}$. Infectious virus (for animal protection experiments) was prepared by infecting cells at a multiplicity of 10 . Medium was removed at $18 \mathrm{~h}$ after infection, and the cells were scraped into a small volume. Cells were sonicated and debris was removed by low speed centrifugation. The supernatant was stored at $-80^{\circ}$ C. The number of infectious virus particles was determined by a plaque assay. Briefly, virus dilutions were plated on monolayers of Vero cells in six well trays. Overlay medium containing $0.5 \%$ methyl cellulose was added. After 3 days, the monolayers were fixed and subsequently stained with Giemsa. Plaques were counted and the titers expresses as pfu per ml.

\section{Design of MEAPs}

The twelve selected epitopes, six B cell epitopes ( $\mathrm{gB} 2_{466-}$ 473, $\mathrm{gC2} 2_{216-223}, \mathrm{gD} 2_{6-18}, \mathrm{gE} 2_{483-491}, \mathrm{gG} 2_{572-579}$ and $\mathrm{gI} 2_{286-}$ ${ }_{295}$ ), four $\mathrm{CD} 4^{+} \mathrm{T}$ cell epitopes (gD2 $21-28, \mathrm{gD} 2_{205-224}$, gD2 $245-259$ and gB2 $2_{162-177}$ ) and two $\mathrm{CD}^{+} \mathrm{T}$ cell epitopes (gD2 $10-20$ and gD2 268-276), which all identified by further experiments (data no shown), were inserted into the extracellular fragment (1-290) of HSV-2 glycoprotein D to construct multi-epitope assembly peptides (MEAPs) by replacing some non-epitope amino acid sequences $[9,12]$. In order to find a homology model for suitable protein structure template, homologous protein sequences were searched using the protein blast method in the PDB database of the United States National Center for Biotechnology Information (NCBI). The results showed that the highest score from 1JMA was about $84 \%$ (30\% protein homology is considered as a condition of homology modeling). So, 1JMA was used as a template for homology modeling of the multi-epitope assembly peptides (MEAPs). The structures the MEAPs were analyzed by Accelrys Discovery Studio and Moe2008 software, only the MEAPs with inserted epitopes independently displayed on the surface were selected as candidate proteins. 
Molecular cloning, expression, and purification of MEAP The gene of a selected MEAP was chemically synthesized (by Jinsite Company, China), and cloned into the sites between BamHIand XhoIof plasmid pET28a $(+)$-sumo to construct a recombinant expression vector of pET28a (+)-sumo-MEAP, and thereafter transformed into E. coli BL21 for expressing a sumo-MEAP fusion protein. The engineered E.coli BL21 was cultured and induced by $1 \mathrm{mM}$ IPTG (Promega) at $25^{\circ} \mathrm{C}$ for $4 \mathrm{~h}$. The induced engineered $E$. coli BL21 was collected by centrifugation, and lysised by ultrasound, the lysis supernatant was harvested by centrifugation, and concentrated by ultrafiltration.

Ni Sepharose 6 Fast Flow (GE Healthcare, Sweden) was suspended by gently rocking the bottle, an adequate amount of slurry was draw into a container through a pipe. The gel was washed with cold PBS $\left(4^{\circ} \mathrm{C}\right)$, and thereafter, mixed with the lysis supernatant containing the expressed sumo-MEAP fusion protein. After oscillation for $30 \mathrm{~min}$ and centrifugation at $500 \mathrm{rpm}$ for $5 \mathrm{~min}$ at room temperature, the supernatant was discarded and the precipitated gel was resuspended with washing buffer and loaded to a column, the gel was washed completely with washing buffer. The proteins were sequentially eluted from the column by gradient imidazole solutions $(25,50,100,150,200$ and $250 \mathrm{mM})$ in Tris- $\mathrm{HCl}(250$ $\mathrm{mM} \mathrm{pH} \mathrm{8.5).} \mathrm{The} \mathrm{eluted} \mathrm{parts} \mathrm{were} \mathrm{collected} \mathrm{for} \mathrm{detec-}$ tion of the purified sumo-MEAP fusion protein by SDSPAGE. The elution fusion protein was dialyzed against Tris- $\mathrm{HCl}(50 \mathrm{mM} \mathrm{pH} 8.0)$, then $50 \mu \mathrm{g}$ of sumo-MEAP was digested with $1 \mu \mathrm{l}$ of sumo proteinase (Biotsith, Suzhou, China) with $2 \mathrm{U} / \mu \mathrm{l}$ at $22^{\circ} \mathrm{C}$ for $16 \mathrm{~h}$. The digestion mixture was loaded on a Ni Sepharose 6 Fast Flow (GE Healthcare, Sweden) column in order to eliminate the sumo and sumo-MEAP that was not digested by sumo proteinase, and then was detected by SDS-PAGE electrophoresis.

\section{Immunization of mice}

Thirty KunMing Mice (4-6 weeks old, male), were randomly divided into three groups. Each group was subcutaneously injected with MEAP, inactivated vaccine and PBS respectively. The immunization program as follows, the first injection with $4 \mu \mathrm{g}$ of the purified MEAP (98\%) emulsified in complete Freund's adjuvant, after 2 weeks, $2 \mu \mathrm{g}$ of the purified MEAP emulsified in incomplete Freund's adjuvant was injected subcutaneously. The third injection was as that of the second. PBS, or inactivated vaccine was injected using the same procedure as MEAP immunization. Serum samples were collected on weeks 2, 3, 4 and 5 weeks after the first injection. Serum samples were separated and stored at $-20^{\circ} \mathrm{C}$ for further assays. Two weeks after the final immunization, the mice were sacrificed, and their spleens removed aseptically for in vitro splenocyte culture. The antigenicity and immunity analysis of the MEAP were performed by EIA. Specific serum IgG antibodies to MEAP were determined with an endpoint EIA using purified MEAP as antigen with $100 \mu \mathrm{l}(2.5 \mu \mathrm{g} / \mathrm{ml})$ in each well as described previously [13]. The titers were expressed as the reciprocal of highest dilution of sera producing ratio values of 2.1. The selected synthesized B cell epitopes were coated as antigens in 96-well plate with 100 $\mu \mathrm{l}(2.5 \mu \mathrm{g} / \mathrm{ml})$ in each well. The antigenicities of B cell epitopes were detected by EIA with antibody to the MEAP. On the other hand, in order to detect the MEAP antigenicity, the MEAP was coated as antigen in 96-well plate with $100 \mu \mathrm{l}(2.5 \mu \mathrm{g} / \mathrm{ml})$ in each well. The MEAP antigenicities against the antibodies to the B, C, D, E, G and I glycoproteins (ABcam) were detected by EIA. All animal work was approved by the Anhui Administrative Committee for Laboratory Animals.

\section{Virus neutralization tests in vitro}

Virus neutralization assay was performed using the procedures described by Cha et al. [14] and Kang et al. [15]. Neutralization antibodies elicited in immunized mice blood from each group was heated at $56^{\circ} \mathrm{C}$ for 30 min to inactivate the complement. A $100 \mu 1$ of the heat-inactivated serum was added into a 96-well-flatbottom microtiter plate for serial dilution (Falcon, Lincoln Park, NJ, USA). A $100 \mu 1$ of $200 \times$ TCID $_{50}$ of the live Sav strain of $\mathrm{HSV}-2$ was added to the plate, sealed and incubated for $18 \mathrm{~h}$ at $40^{\circ} \mathrm{C}$. Then $100 \mu 1$ of $5 \times 10^{3}$ viable Vero cells were added to each well and incubated at $37^{\circ} \mathrm{C}$ for 5 days. The serum dilution factor that neutralized $50 \%$ of the virus was determined as the titer. The TCID $_{50}$ was $0.69 \mathrm{pfu}$ as determined by the procedure described by Reed and Muench [16]. Each serum sample was assayed in triplicate.

\section{Cytokine profiling}

The vaccinated mice, from the above, were sacrificed to recover splenocytes 2 weeks after final immunization. The splenocytes were incubated in 96-well flat-bottomed microtiter plates $\left(100 \mu 1 /\right.$ well of $5 \times 10^{6} \mathrm{cell} / \mathrm{ml}$ in RPMI 1640 medium with 10\% FCS). The MEAP protein was added at a final concentration of $10 \mu \mathrm{g} / \mathrm{ml}$. After incubation for $72 \mathrm{~h}$, the splenocyte culture supernatants were collected for cytokine detection. The presence of the cytokines IL-2, IL-4 and IFN- $\gamma$ in supernatant from the splenocytes cultured from vaccinated mice was examined using commercial murine cytokine EIA kits (Biosource USA), following the manufacturer's instructions. 


\section{Cytotoxic T lymphocyte (CTL) assay}

The CTL assay was performed as described earlier [17]. Briefly, mixed splenocytes from 10 mice in each group were stimulated with the MEAP protein $(10 \mu \mathrm{g} / \mathrm{ml})$ in 1 $\mathrm{ml}$ of RPMI 1640 medium with 10\% FCS in 24-well microplates at $37^{\circ} \mathrm{C}$ for 6 days. Target cells (NIH 3T3 cells) were prepared by infection with the HSV-2 Sav for $20 \mathrm{~h}$ before the assay. The target cells were distributed into triplicate wells of a 96-well plate $\left(5 \times 10^{3}\right.$ cells per well) and the ratio of effector to target cells was adjusted to 50: 1. The effector and target cells were mixed and incubated at $37^{\circ} \mathrm{C}$ for $6 \mathrm{~h}$ before the supernatant was collected. Lactate dehydrogenase (LDH) activity released into the culture medium was measured with a cytotoxicity assay kit (Promega, USA), according to the manufacturer's instructions.

\section{Animal protection experiments}

The mice ( 8 weeks old, BALB/c, female), 10 each group for three groups, were immunized with $4 \mu \mathrm{g}$ of the MEAP, inactivated vaccine and PBS as control, were challenged with the Sav strain. At 2 weeks after the final vaccination was injected, the anesthetized mice were challenged with the Sav strain. In order to synchronize the estrus cycle at the progesterone-dominated stage, the immunized and sham-immunized mice were subcutaneously injected with $2 \mathrm{mg}$ of progesterone (Jinsite, China) in $50 \mu 1$ of $\mathrm{H}_{2} \mathrm{O}$ per mouse. At 5 days after the administration of progesterone, the mice of all groups were infected with the Sav strain into vagina and external genital skin. One hour prior to infection, the vaginal closure membrane was ruptured with a saline-moistened cotton swab. The swab was inserted into vagina, twisted back and for five times, then removed and wiped over the external genitalia. To ensure infection, a virus application was repeated $1 \mathrm{~h}$ later. The infected mice were examined daily for vaginal inflammation, neurological illness, and death, and then were scored in steps 1-5 depending on the severity of disease as described by the reported papers $[17,18]$. Vaginal washings were collected on days 1, 2, 3, and 4 post inoculation after the intravaginal challenge with pipetting $100 \mu \mathrm{l}$ of PBS into the vaginal cavity. The samples were stored at $-20^{\circ} \mathrm{C}$ until used. They were added to $1 \mathrm{ml}$ of the media, and were subsequently infected and titered on Vero cell monolayers by using a plaque assay. Viral neutralization titers in the vaginal secretion samples of the day 4 post inoculation were measured using a micro-neutralization assay described above. The immunized mice were intravaginal challenged with lethal dosages $\left(5 \times 10^{6}\right.$ pfu: $\left.500 \mathrm{LD}_{50}\right)$ of the Sav strain. The vaginal external diseases were examined daily for signs of inflammation and measured mean daily lesion scores on Day 1-14 following infection. The severity of the primary disease was assessed by the lesion scoring system $[19,20]$. In addition, survival of the vaccinated mice challenged with the Sav strain were daily measured and summarized.

\section{Statistical analysis}

Data were expressed as means \pm standard error (SE). Student's $t$-test or analysis of variance (ANOVA) was used for determine the differences among the groups, using the SPSS software package (version 16.0, SPSS Inc., Chicago, IL, USA). A value of $P<0.05$ was considered statistically significant.

\section{Results}

\section{Prediction of epitopes independency of MEAP}

The twelve epitopes, including six $\mathrm{B}$, four $\mathrm{CD} 4^{+} \mathrm{T}$ and two $\mathrm{CD} 8^{+} \mathrm{T}$ cell epitopes, were used for construction of the MEAPs. The six B (gB2 $2_{466-473}, \mathrm{gC} 2_{216-223}, \mathrm{gD} 2_{6-18}$, gE2 $2_{483-491}, g G 2_{572-579}$ and gI2 286-295), four CD4 ${ }^{+} \mathrm{T}$ (gD2 21-28, gD2 205-224, gD2 245-259 and gB2 162-177) and two $\mathrm{CD}^{+} \mathrm{T}$ cell epitopes (gD2 $10-20$ and $\left.\mathrm{gD} 2_{268-276}\right)$, were inserted into the extracellular fragment (1-290) of HSV2 glycoprotein $\mathrm{D}$ to construct multi-epitope assembly peptides (MEAPs) by replacing some non-epitope amino acid sequences. Three-dimensional structures of the designed 14 MEAPs were predicted for screening the MEAP in which all the $B$ cell epitopes independently displayed on the surface of the MEAP. The MEAP selected for preparation by genetic engineering was shown in Figure 1 and Figure 2.

\section{Expression and purification of sumo-MEAP}

The gene of the selected MEAP was synthesized chemically and cloned into plasmid pET28a $(+)$-sumo, the recombinant plasmid pET28a (+)-sumo-MEAP was confirmed by DNA sequencing and was transferred into $E$. coli BL21, the expression of recombinant protein was induced by IPTG and screened by SDS-PAGE analysis. Due to a sumo tag located at the N-terminal of the MEAP, the molecular weight of expressed sumo-MEAP fusion protein was approximately $40 \mathrm{kD}$. The expressed sumo-MEAP was about $20 \%$ of the total bacterial proteins and largely existed in soluble form. The results indicated that the recombinant protein sumo-MEAP was successfully expressed. The sumo-MEAP was purified by $\mathrm{Ni}$ Sepharose 6 Fast Flow and detected by SDS-PAGE. The sumo-MEAP was predominantly contained in the elution of $100 \mathrm{mM}$ imidazole. In order to remove the sumo, sumo-MEAP was digested by sumo proteinase at $22^{\circ} \mathrm{C}$ for $2 \mathrm{~h}$, then purified by Ni Sepharose 6 Fast Flow, the collected eluent was detected by SDS-PAGE (Figure 3).

\section{Anti-MEAP IgG antibody titers in the sera of immunized mice}

All the mice immunized with the MEAP produced the antibodies to the MEAP at the titer from 1: 400 to 1: 

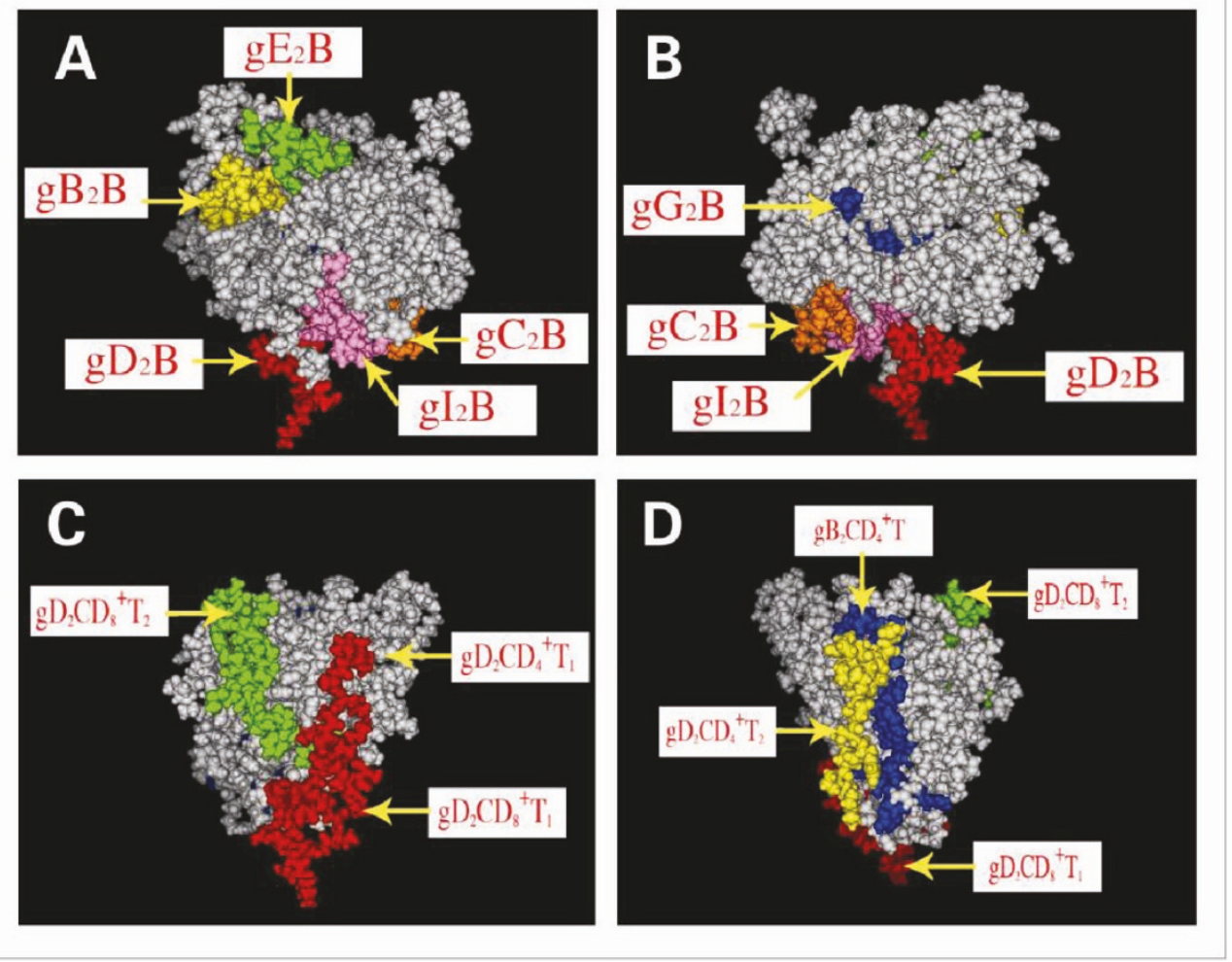

Figure 1 Three-dimensional of epitopes of MEAP.1JMA was used as a template for homology modeling of the multi-epitope assembly peptides (MEAPs). The three-dimensional structures of the MEAP were analyzed by software algorithm Moe2008, while the three-dimensional of epitopes were depicted as follows: Three-dimensional B cell epitopes can be seen in A and B, and A is the obverse side opposite to B. Threedimensional $\mathrm{CD}^{+} \mathrm{T}$ and $\mathrm{CD} 8^{+} \mathrm{T}$ cell epitopes can be seen in $\mathrm{C}$ and $\mathrm{D}$, and $\mathrm{C}$ is the obverse side opposite to $\mathrm{D}$.

10000. The highest titer of antiserum to the MEAP was selected to confirm the immunity of the B cell epitopes by EIA using B cell epitopes as antigens. Also, the MEAP was used as the antigen to detect the corresponding antibodies against gB2, gC2, gE2, gG2, gI2 and gD2 by EIA. All B cell epitopes, except gG2 ${ }_{572-579}$, could react with the antiserum against the MEAP, and the MEAP could react with all the antibodies against the six glycoproteins, but only weakly with the antibodies against gG2 (Table 1). The results indicated that the MEAP had good antigenicity and immunity, and implied that the five $\mathrm{B}$ cell epitopes, $\mathrm{gB} 2_{466-473}, \mathrm{gC} 2_{216-223}, \mathrm{gD} 2_{6-}$ 18, gE2 $483-491$ and gI2 286-295, were displayed on the surface of the MEAP molecule, which was consistent with the prediction. Mice were immunized with the MEAP, inactivated vaccine and PBS by the subcutaneously injection. Serum samples (10 mice per group) were collected at various time points, and endpoint antibody titers against HSV-2 were determined by EIA. The MEAP, inactivated Sav strain and PBS were used to coat in EIA. The result was shown in Figure 4.
Virus neutralization activity of antisera

Serum samples collected on day 14 were evaluated for their ability to neutralize HSV-2 by serum neutralizing assay. Virus-neutralization activity of the antisera against MEAP, inactivated vaccine and PBS were tested by $50 \%$ plaque reduction assay. The neutralizing titer of the antiserum from the mice, which were immunized with the MEAP, had a neutralizing antibody titer of 1/1208. The neutralization titers of antiserum form mice immunized with inactivated vaccine is $1 / 564$. The mice antisera from the PBS-vaccinated mice showed little or no neutralizing activity.

Th1-type and Th2-type cytokine responses were analyzed in immunized mice

The splenocyte culture supernatant was harvested at 72 $\mathrm{h}$ after stimulation to determine the production of different cytokines (IL-2, IL-4 and IFN- $\gamma$ ). Splenocytes from the mice immunized with MEAP induced the highest level of IL-4 $(311.23 \pm 6.57 \mathrm{pg} / \mathrm{ml})$ among the three groups. The levels of IL- 2 and IFN- $\gamma$ were 149.97 


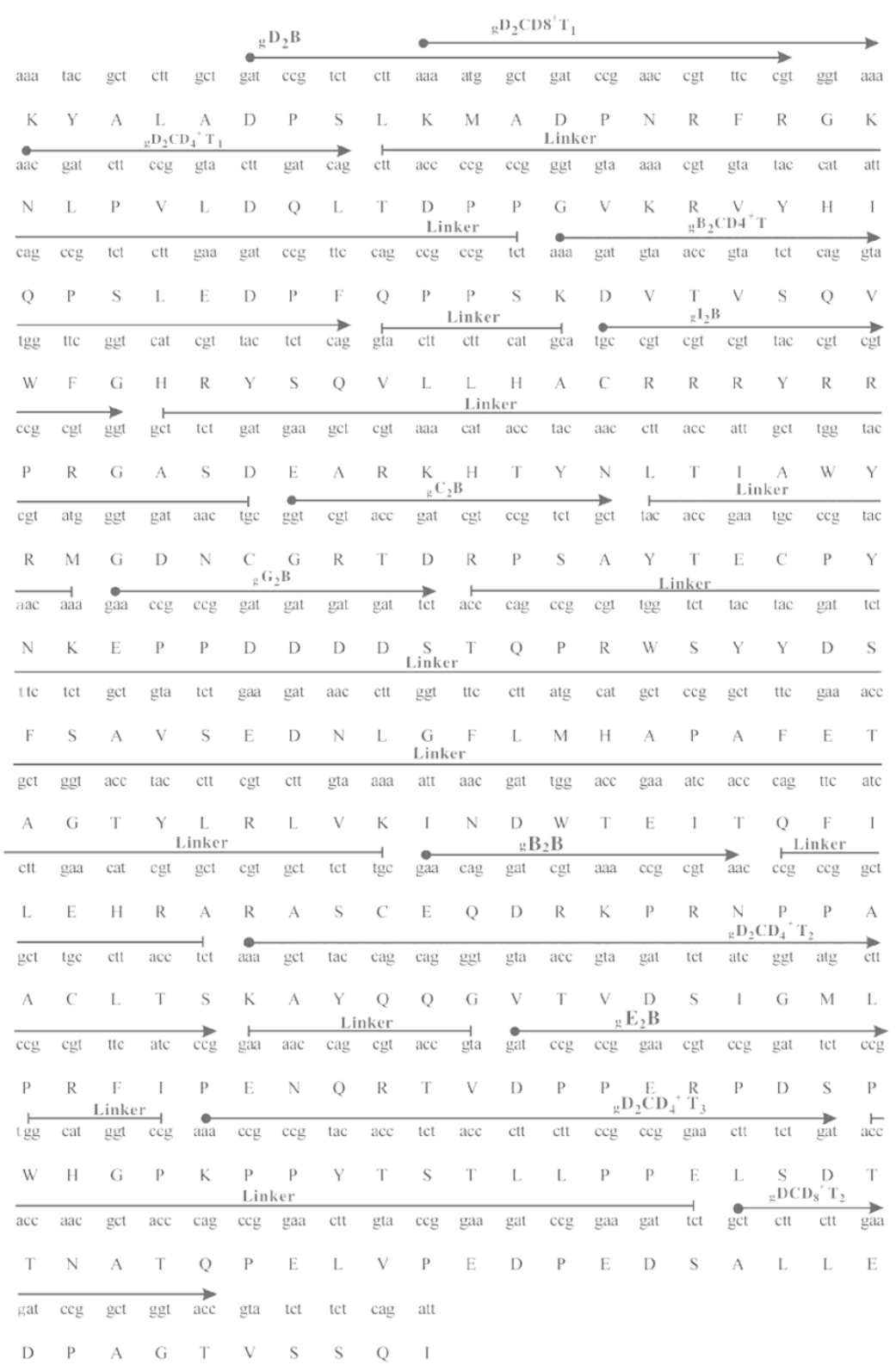

Figure 2 Design and construction a multi-epitope assembly peptide (MEAP). The MEAP were constructed from six B cell epitopes (gB2466-

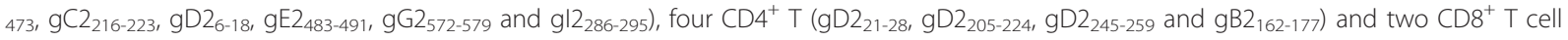
epitopes ( $\mathrm{gD}_{10-20}$ and $\mathrm{gD2} 268-276$ ) with some non-epitope amino acid sequences of the extracellular fragment (1-290) of HSV-2 glycoprotein D as spacers between epitopes.

\pm 7.53 and $169.11 \pm 6.96 \mathrm{pg} / \mathrm{ml}$. This result demonstrated that splenocyte from the mice immunized with MEAP induced predominantly Th-2 (IL-4)-type cellular immune response, because the levels of Th-2 (IL-4)-type cytokines were higher than those of Th1 (IL-2 and IFN$\gamma$ )-type cytokines. Splenocyte from the mice immunized with the inactivated vaccine made only small amounts of IL-4 (119.21 $\pm 6.98 \mathrm{pg} / \mathrm{ml})$; nevertheless, they induced high levels of IFN- $\gamma(219.33 \pm 6.99 \mathrm{pg} / \mathrm{ml})$ and IL-2 $(181.70 \pm 17.43 \mathrm{pg} / \mathrm{ml})$. Splenocyte from the mice that received PBS did not produce any significant cytokine titers (Figure 5).

\section{Cytokine T lymphocytes (CTL) among splenocyte stimulated with MEAP in vitro}

The highest level of CTL activity was observed in the group of splenocyte treatment with MEAP in cytotoxicity assay, followed by the groups inoculated with the inactivated vaccine and PBS. The mean CTL activity of MEAP treatment group, inactivated vaccine treatment 


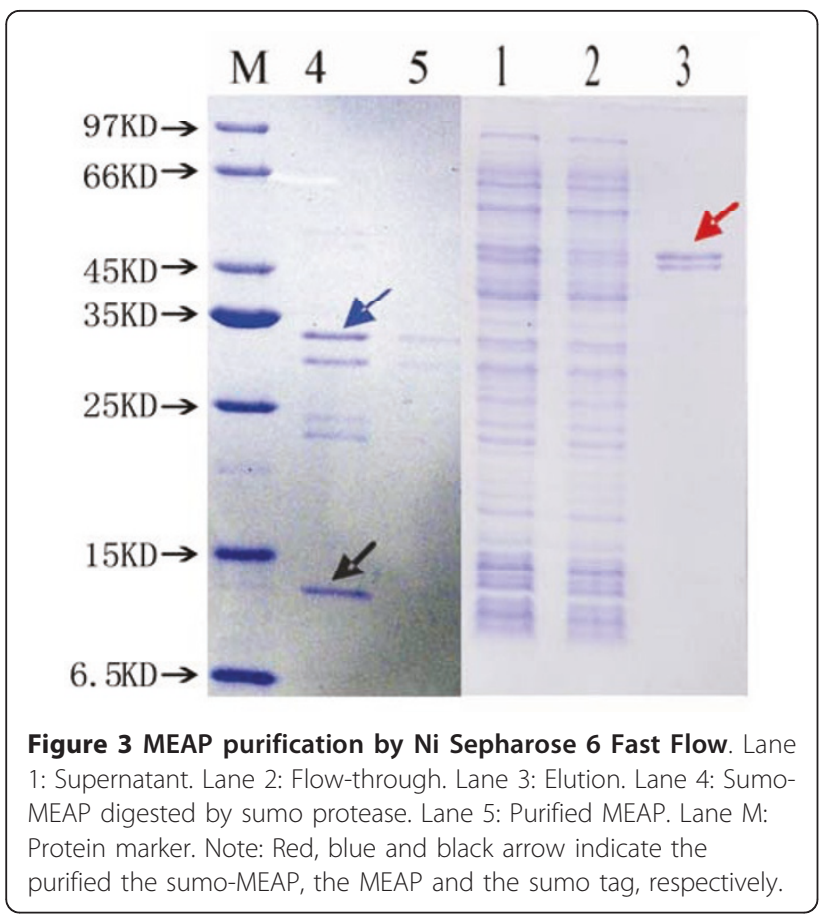

group and PBS group was $59.94 \pm 5.43 \%, 18.21 \pm 1.43 \%$ and $12.21 \pm 1.51 \%$, respectively. The CTL responses from the group immunized with MEAP were significantly higher than those from the groups given the inactivated vaccine or PBS $(P<0.01)$ (Figure 6$)$.

Table 1 Antigenicities of the MEAP with antibodies to B, C, D, E, G and I glycoprotein of HSV-2 and antigenicities of some B cell epitopes with antibodies to the MEAP

\begin{tabular}{lll}
\hline No. & Antibodies & EIA OD $(\mathbf{4 5 0} \mathbf{n m})$ Value \\
\hline gD2 $6-18$ & Ab to MEAP & $1.125 \pm 0.041$ \\
gB2 $466-473$ & Ab to MEAP & $0.977 \pm 0.039$ \\
gC2 $216-223$ & Ab to MEAP & $0.899 \pm 0.031$ \\
gE2 $483-491$ & Ab to MEAP & $1.102 \pm 0.040$ \\
gG2 $572-579$ & Ab to MEAP & $0.182 \pm 0.011$ \\
gl2 $286-295$ & Ab to MEAP & $0.792 \pm 0.029$ \\
MEAP & Ab to gD2 & $1.099 \pm 0.037$ \\
MEAP & Ab to gB2 & $0.986 \pm 0.031$ \\
MEAP & Ab to gC2 & $0.893 \pm 0.029$ \\
MEAP & Ab to gE2 & $1.013 \pm 0.041$ \\
MEAP & Ab to gG2 & $0.201 \pm 0.011$ \\
MEAP & Ab to gl2 & $0.876 \pm 0.031$ \\
Control 1 & Ab to MEAP & $0.116 \pm 0.011$ \\
Control 2 & Ab to gD2 & $0.121 \pm 0.010$ \\
\hline
\end{tabular}

The selected synthesized B cell epitopes were coated as antigens. The antibodies to the MEAP reacted with the epitopes. The antigenicities were detected by EIA. The MEAP was coated as an antigen. The antibodies to the $B$ $C, D, E, G$ and I glycoproteins (ABcam) reacted with the MEAP, and the results can be seen in the Table. No. represents the number of the peptides and the MEAP. Control 1 is a peptide other from the HSV-2 glycoproteins. Control 2 is a antibody other than antibodies to the B, C, D, E, G and I glycoproteins of HSV-2. The O.D. mean values (at $450 \mathrm{~nm}$ ) are shown in the Table.

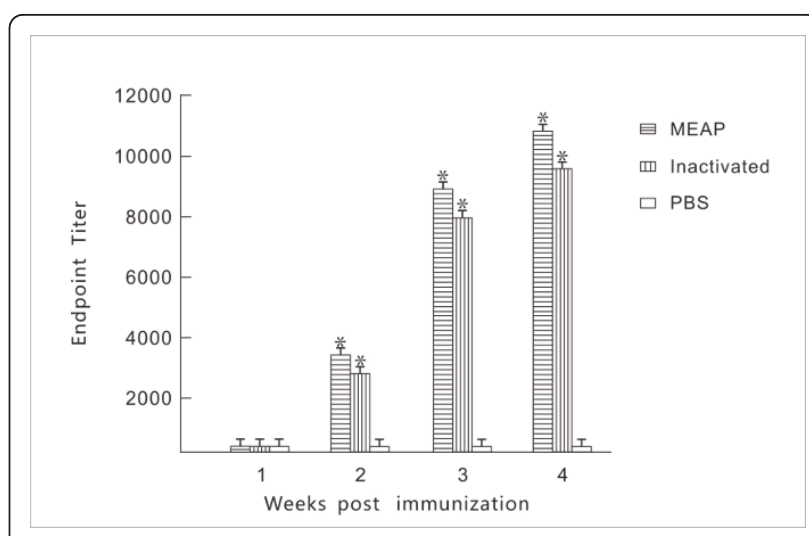

Figure 4 Antibody response in mice. Group of BALB/C mice were immunized with the MEAP, inactivated Sav vaccine and PBS. Serum samples (10 mice per group) were collected at various time points, and endpoint antibody titers against HSV-2 were determined by EIA. All the measurements were made in triplicate, with the mean \pm standard error (SE) shown. $P<0.05$ compared with groups immunized with PBS or inactivated vaccine.

\section{Animal protection experiments}

The immunized mice were intravaginal challenged with lethal dosages $\left(5 \times 10^{6}\right.$ pfu: $\left.500 \mathrm{LD}_{50}\right)$ of the Sav strain. The vaginal external diseases were examined daily for signs of inflammation and measured mean daily lesion scores on day 1-14 following infection. The severity of the primary disease was assessed by the lesion scoring system $[19,20]$. The negative vaccination developed severe diseases and had no effect after 3 days, but the MEAP immunized groups were completely protected from death. The inactivated vaccine immunized mice also showed a high protection level of the lesion score 1 stage from day 4 . These results indicated that the

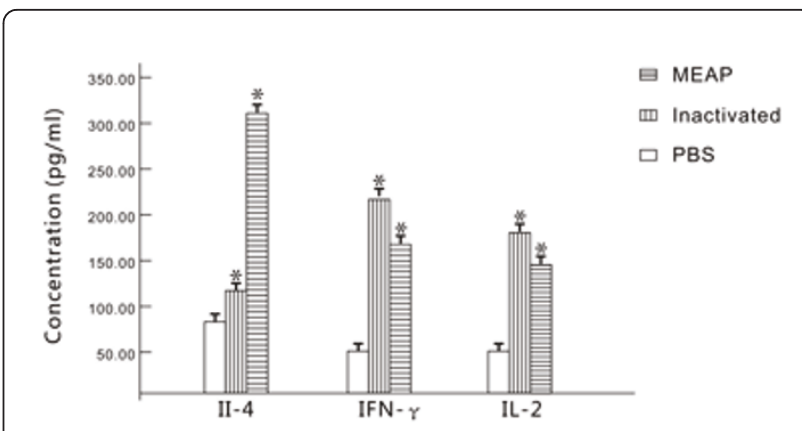

Figure 5 Cytokine detection in splenocyte culture supernatants from vaccinated mice. Splenocyte samples (10 mice per group) isolated from the spleens of vaccinated mice on day 14 after the last immunization against the MEAP. After $72 \mathrm{~h}$, the supernatants were collected to examine the levels of the Th1- type cytokine IL-2, IFN- $\gamma$ and the Th2-type cytokine IL-4 using commercially available murine cytokine EIA kits. All the measurements were made in triplicate, with the mean \pm standard error (SE) shown. $P<0.05$ compared with groups immunized with PBS or inactivated vaccine. 


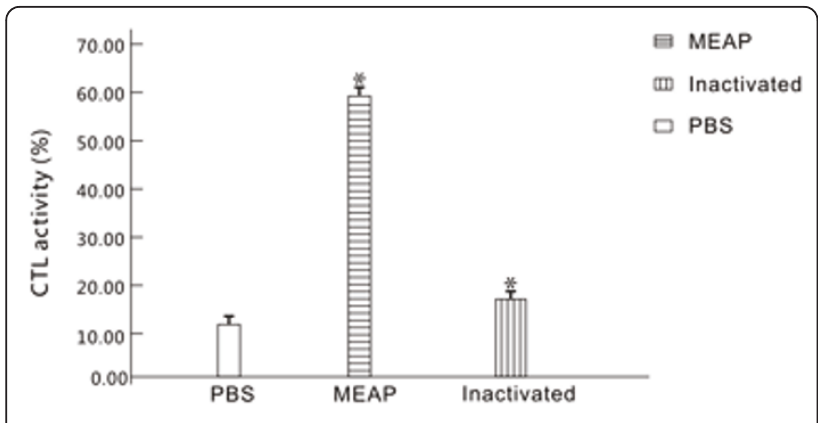

Figure 6 CTL activity of splenocytes from vaccinated mice 2 weeks after the last immunization. Splenocyte samples (10 mice per group) isolated from the vaccinated mice were stimulated with the MEAP. After 6 days the CTL activities were detected using a cytotoxicity assay kit by measurement of the LDH released. All the measurements were made in triplicate, with the mean \pm standard error (SE) shown. $P<0.05$ compared with groups immunized with PBS or inactivated vaccine.

immunized mice with the MEAP and the inactivated vaccine had protective effect on HSV-2 infection.

The MEAP and inactivated vaccine immunized groups completely survived during experiments, while the PBS vaccination mice developed severe diseases with all the mice dead after 5 days post the infection (Figure 7).

Virus multiplication was also observed in the genital tracts of all immunized mice on 4th days post infection. The virus was isolated in vaginal tracts with cotton swabs from the immunized mice. Virus titers in the immunized mice on days 1, 2, 3 and 4 post intravaginal infection were significantly reduced than that of the negative control (PBS). Also, each vaccinated group demonstrated that the viral titers decreased with time. On day 1 , viral titer of the MEAP immunized mice decreased 4.76 fold than that of the PBS negative control group. Viral titers of the inactivated vaccinated mice were nearly similar to the MEAP immunized mice. On day 4 , the virus titer decreased about 4.07 times in the inactivated vaccinated

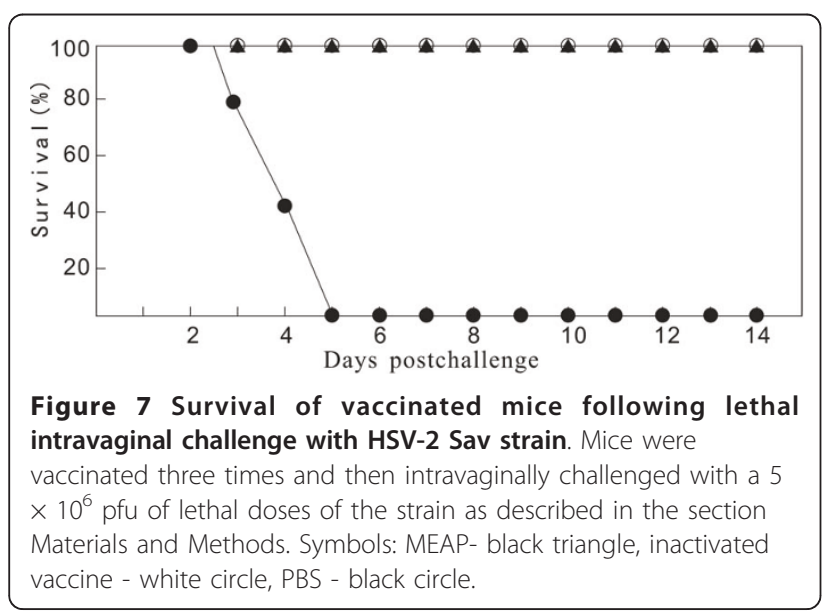

mice and 4.18 times in the MEAP immunized mice than that of the PBS control. These results indicated that virus in MEAP immunized mice and inactivated vaccinated mice were cleared more rapidly from the genital tracts of mice than in PBS treatment mice $(P<0.05)$, and the MEAP has better protective effect against the HSV-2 infection with lethal dosage.

\section{Discussion}

The potential advantages of an epitope based vaccine are as follows. First, epitope based vaccines represent a powerful approach to induce a specific immune response against the selected epitope (s), avoiding the side effects of other unfavorable epitopes in the complete antigen [21]. Second, epitope based vaccine has other considerable advantages, including increased safety, the opportunity to engineer the epitopes rationally for increased potency and breadth, and the ability to focus the immune response on conserved epitopes [22]. Moreover, the epitope based vaccine approach has been shown to be successful in various infectious diseases, such as Neisseria meningitides infection, and tuberculosis [23]. In this study, an epitope based vaccine was proposed as a possible candidate vaccine with a remarkable ability to induce protection against HSV-2 infection.

B cell antigenic epitopes from HSV-2 glycoproteins were screened using three software algorithms: Biosun, DNAstar, and Antheprot algorithms. The common results from software were used as candidate epitopes of $\mathrm{B}$ cell epitopes. In this study, six B cell epitopes (gB2 466 473, $\mathrm{gC2}_{216-223}, \mathrm{gD} 2_{6-18}, \mathrm{gE} 2_{483-491}, \mathrm{gG} 2_{572-579}$ and $\mathrm{gI} 2_{286-}$ 295) were predicted and identified as epitopes (data no shown), which have not been reported. $\mathrm{CD}^{+} \mathrm{T}$ cell epitopes were predicted successively by the APC software algorithm, the lysosome hydrolysis cleavage site software algorithm and the TAP software algorithm. The results of the APC software algorithm provided candidate peptides for the lysosome hydrolysis cleavage site software algorithm. Similarly, the results of the lysosome hydrolysis cleavage site software algorithm provided candidate peptides for the TAP bind software. Some $\mathrm{CD} 4^{+} \mathrm{T}$ cell immunodominant epitopes, which had higher affinity to TAP than ICP47, were screened as candidate $\mathrm{CD} 4^{+} \mathrm{T}$ cell epitopes [24]. Four of them (gD2 21-28, gD2 205-224, gD2 $245-259$ and $g B 2_{162-177}$ ) were identified as epitopes (data no shown), which have not been reported. Here, epitope-prediction program SYFPEITHI, NetMHC and MHCPred were used to analyze the sequence of gD2 according to their different algorithms. Two $\mathrm{CD} 8^{+} \mathrm{T}$ cell immunodominant epitopes, gD2 $2_{10-20}$ and gD2 $2_{268-276}$, were predicted and identified as epitopes (data no shown) [25], which have not been reported.

The gD2, gB2, gC2, gE2, gG2 and gI2 of HSV-2 are largely exposed structural glycoprotein, which have B- 
cell and T-cell epitopes and are major immunogenic antigen [26]. Ideally, epitope based vaccines should contain both B cell epitopes, which are essential for the protective antibody response, and T cell epitope (CTL epitopes, Th epitopes) that will serve to induce CTL and Th immune responses. Therefore, in the present study, we selected six B cell epitopes ( $\mathrm{gB} 2_{466-473}, \mathrm{gC2} 2_{216-223}$, gD2 $6-18, g E 2_{483-491}, g G 2_{572-579}$ and gI2 $\left.286-295\right)$, four CD4 ${ }^{+}$ $\mathrm{T}$ cell epitopes (gD2 21-28, gD2 205-224, gD2 $245-259$ and gB2 162-177) and two CD8 ${ }^{+} \mathrm{T}$ cell epitopes (gD2 $10-20$ and $\mathrm{gD} 2$ 268-276) from HSV-2 glycoproteins to construct an epitope based vaccine (MEAP). These confirmed epitopes were all inserted into the extracellular fragment (1-290) of HSV-2 glycoprotein D to construct multi-epitope assembly peptides (MEAPs) by replacing some non-epitope amino acid sequences. Three-dimensional structures of the 14 MEAPs were predicted by the software algorithms of Accelrys Discovery Studio and Moe2008 in order to screen a MEAP. All epitopes, especially the B cell epitopes, independently displayed on the surface of the MEAP [12,27,28].

HSV-2 specific IgG response was detected in the sera of mice immunized with MEAP after three boosts, as well as in the mice immunized with inactivated vaccine. This suggests that the MEAP protein and inactivated vaccine were both capable of inducing a significant humoral response in mice. Studies have demonstrated that neutralizing antibodies are important for protection against HSV-2 infection and titers $\geq 1: 10$ are considered to be indicative of protective immunity [29]. The mean neutralization titer in the mice immunized with MEAP was 1208 after the third boost, and these titers were higher than those induced by the inactivated vaccine $(P$ $<0.05)$. Importantly, these antibody titers were sufficient to protect mice against HSV-2 infection, which indicates the potential of MEAP as a vaccine against HSV-2.

In addition, Cell-mediated immunity plays an important role in efficient protection. The results of cytokine assay and CTL activity indicated that CTL activity and the level of Th2-type cytokines (IL-4) produced in the culture supernatant from mice vaccinated with MEAP was significantly higher than in the groups given inactivated vaccine and $P B S$. This demonstrated that the multi-epitope vaccine, MEAP, constructed in this study stimulated intensive cellular immunoreaction. Specific CTL are critical in the recovery from infection and the clearance of HSV-2, and the Th2 immune response against HSV-2 is also protective [30], the remarkably increased CTL activity and IL-4 production in the MEAP group may indicated that the MEAP has a higher degree of protection effect.

Challenge experiments showed that the mice that received the MEAP or the inactivated vaccine were completely protected against HSV-2 infection, even though the mean neutralizing antibody titer before challenge of the group vaccinated with the MEAP was lower than that of the group given the inactivated vaccine. This high level of protection, also, is usually related to the MEAP specific neutralizing antibodies [31]. However, we observed high levels of CTL activity in the splenocytes of the mice immunized with the MEAP. This may be contributed to the high levels of protection in the mice immunized with the MEAP, because CTL activity by the $\mathrm{T}$ cells was found also to be an essential determinant of protective immunity against HSV-2 [32].

\section{Conclusions}

We have constructed a recombinant multi-epitope assembly peptide (MEAP) from HSV-2 using epitope based vaccine strategies. These results indicated that MEAP was capable of inducing remarkable humoral and cellular immune responses and provided complete protection against lethal challenge in mice. These demonstrate that the MEAP may be a promising subunit vaccine candidate for the prevention of HSV-2 infection. The present study also provided useful information for the further development of multi-epitope vaccine for human.

\section{Acknowledgements}

We acknowledge grant support from the No. 20080431414 of the China Postdoctoral Science Foundation, No. BK 2008068 of the China Jiangsu National Science Foundation, 2006AA02A226 of the National High-Tech R \& D Program of China (863 Program), No. KJ2010A221 of the Anhui Education Department Key Program, and No. BE2010603 of Jiangsu Social Development Foundation.

\section{Author details}

'Huadong Research Institute for Medicine and Biotechniques, Nanjing, 210002, China. ${ }^{2}$ School of Life Science and Technology, China Pharmaceutical University, Nanjing, China, 210009. ${ }^{3}$ Department of Pharmacy, Anhui University of Traditional Chinese Medicine, Hefei, China, 230038.

${ }^{4}$ Department of Immunology and Microbiology, Anhui Agriculture University, Hefei, China, 230038.

\section{Authors' contributions}

XW responsible for conducting experiment and writing the manuscript. GX responsible for conducting some of experiment and writing some of the manuscript. $J L$ was responsible for epitopes prediction and tested antibodies activity in vitro, and DY was responsible for cytokine profiling. WG was responsible for cytotoxic T lymphocyte assay. MP responsible for anima protection experiments, YL and JL responsible for conducting experimental design and conducting experiment. All authors read and approved the final manuscript.

\section{Competing interests}

The authors declare that they have no competing interests.

Received: 13 December 2010 Accepted: 16 May 2011

Published: 16 May 2011

\section{References}

1. L Corey, Challenges in genital herpes simplex virus management. J Infect Dis. 186(Suppl. 1):S29-S33 (2002)

2. DM Koelle, L Corey, Recent progress in herpes simplex virus immunobiology and vaccine research. Clin Microbiol. 16, 96-113 (2003). doi:10.1128/CMR.16.1.96-113.2003 
3. RJ Whitley, B Roizman, Herpes simplexvirus infections. Lancet. 35, 1513-1518 (2001)

4. J Akhtar, D Shukla, Viral entry mechanisms: cellular and viral mediators of herpes simplex virus entry. FEBS J. 276, 7228-36 (2009). doi:10.1111/j.17424658.2009.07402.x

5. T Gianni, C Forghieri, G Campadelli-Fiume, The herpesvirus glycoproteins B and $\mathrm{H} / \mathrm{L}$ are sequentially recruited to the receptor bound gD to effect membrane fusion at virus entry. Proc Natl Acad Sci. 103, 14572-7 (2006). doi:10.1073/pnas.0606127103

6. PE Pertel, A Fridberg, ML Parish, PG Spear, Cell fusion induced by herpes simplex virus glycoproteins $\mathrm{gB}, \mathrm{gD}$, and $\mathrm{gH}$-gL requires a $\mathrm{gD}$ receptor but not necessarily heparan sulfate. Virology. 279, 313-32 (2001). doi:10.1006/ viro.2000.0713

7. C Krummenacher, VM Supekar, JC Whitbeck, E Lazear, SA Connolly, RJ Eisenberg, GH Cohen, DC Wiley, A Carfi, Structure of unliganded HSV gD reveals a mechanism for receptor-mediated activation of virus entry. EMBO J. 24, 4144-4153 (2005). doi:10.1038/sj.emboj.7600875

8. PE Pertel, A Fridberg, ML Parish, Spear PG Cell fusion induced by herpes simplex virus glycoproteins $\mathrm{gB}, \mathrm{gD}$, and $\mathrm{gH}-\mathrm{gL}$ requires a $\mathrm{gD}$ receptor but not necessarily heparan sulfate. Virology. 279, 313-324 (2001). doi:10.1006/ viro. 2000.0713

9. J Sollne, R Grohmann, R Rapberger, P Perco, A Lukas, B Mayer, Analysis and prediction of protective continuous B-cell epitopes on pathogen proteins. Immunome Research. 4, 1 (2008). doi:10.1186/1745-7580-4-1

10. G Mezo, B Dalmadi, I Mucsi, S Bosze, E Rajnavölgyl, F Hudecz, Peptide Based Vaccine Design: Synthesis and Immunological Characterization of Branched Polypeptide Conjugates Comprising the 276-284 Immunodominant Epitope of HSV-1 Glycoprotein D. Journal of Peptide Science. 8, 107-117 (2002). doi:10.1002/psc.373

11. H Ghiasi, S Cai, GC Perng, AB Nesburn, SL Wechsler, Both CD4+ and CD8+ $\mathrm{T}$ cells are involved in protection against HSV-1 induced corneal scarring. $\mathrm{Br}$ J Ophthalmol. 84, 408-412 (2008)

12. AP Haste, M Nielsen, O Lund, Prediction of residues in discontinuous B-cell epitopes using protein 3D structures. Protein Science. 15, 2558-2567 (2006). doi:10.1110/ps.062405906

13. R Kaur, G Sachdeva, S Vrati, Plasmid DNA immunization against Japanese encephalitis virus: immunogenicity of membrane-anchored and secretory envelope protein. J Infect Dis. 185, 1-12 (2002). doi:10.1086/338015

14. H Cha SC. Kang, SY Lee, GJ Park, HH Lee, Expression of the HSV1glycoprotein B gene in insect cells infected by HCNPV recombinant. J Microbiol Biotechnol. 10, 355-362 (2000)

15. H Kang, SC Cha, YJ Han, IH Park, MJ Lee, SM Byun, HH Lee, High level production of glycoprotein protein $\mathrm{H}$ of the HSV-1(F) using HcNPV vector system. J Biochem Mol Biol. 33, 483-492 (2000)

16. HJ Geerligs, H Clemens, M Kocken, JW Drijfhout, WJ Weijer, W Bloemhoff, JB Wilterdink, W Welling, S Welling-Wester, Virus neutralizing activity induced by synthetic peptides of glycoprotein D of Herpes simplex virus type 1 , selected by their reactivity with hyperimmune sera from mice. Journal of General virology. 71, 1767-1774 (1990). doi:10.1099/0022-1317-71-8-1767

17. SC Cha, YS Kim, JK Cho, J Cho, SY Kim, H Kang, MH Cho, HH Lee, Enhanced protection against HSV lethal challenges in mice by immunization with a combined HSV-1 glycoprotein B: H: L gene DNAs. Virus Res. 86, 21-31 (2002). doi:10.1016/S0168-1702(02)00037-0

18. S Khattar, KPL Collins, SK Samal, Immunization of cattle with recombinant Newcastle disease virus expressing bovine herpesvirus-1 (BHV-1) glycoprotein $\mathrm{D}$ induces mucosal and serum antibody responses and provides partial protection against BHV-1. Vaccine. 28, 3159-70 (2010). doi:10.1016/j.vaccine.2010.02.051

19. HH Lee, SC Cha, DJ Jang, JK Lee, DW Choo, YS Kim, HS Uh, SY Kim, Immunization with combined HSV-2 glycoproteins B2: D2 gene DNAs: protection against lethal intravaginal challenges in mice. Virus Genes. 25, 179-88 (2002). doi:10.1023/A:1020113902834

20. A Simmons, AA Nash, Zosteriform spread of herpes simplex virus as a model of recrudescence and its use to investigate the role of immune cells in prevention of recurrent disease. J Virol. 52, 816-21 (1984)

21. T Saarne, L Kaiser, H Gronlund, O Rasool, G Gafvelin, M Van, Hage-Hamsten, Rational design of hypoallergens applied to the major cat allergen Fel $\mathrm{d} 1$. Clin Exp Allrgy. 35, 657-663 (2005). doi:10.1111/j.1365-2222.2005.02234.x

22. A Sette, J Fikes, Epitope-based vaccines: an update on epitope identification, vaccine design and delivery. Curr Opin Immunol. 15, 461-470 (2003). doi:10.1016/50952-7915(03)00083-9
23. AS Groot De, J McMurry, J Franco, D Rivera, M Kutzler, D Weiner, B Martin, Developing an epitope-driven tuberculosis (TB) vaccine. Vaccine. 23, 2121-2131 (2005). doi:10.1016/j.vaccine.2005.01.059

24. JS Burgos, E Serrano-Saiz, I Sastre, F Valdivieso, ICP47 mediates viral neuroinvasiveness by induction of TAP protein following intravenous inoculation of herpes simplex virus type 1 in mice. J Neurovirol. 12, 420-7 (2006). doi:10.1080/13550280601009546

25. B Wang, K Yao, G Liu, F Xie, F Zhou, Y Chen, Computational Prediction and Identification of Epstein-Barr Virus Latent Membrane Protein 2A AntigenSpecific CD8 ${ }^{+}$T-cell. Cellular \& Molecular Immunology. 6, 97-103 (2009). doi:10.1038/cmi.2009.13

26. R Kaur, S Vrati, Development of a recombinant vaccine against Japanese encephalitis. J Neurovirol. 9, 421-431 (2003)

27. YX Huang, YL Bao, SY Guo, Y Wang, CG Zhou, YX Li, Pep-3D-Search: a method for B-cell epitope prediction based on mimotope analysis. BMC Bioinformatics. 9, 5381-5387 (2008)

28. J Ponomarenko, W Huynh-Hoa Bui, N Li, P Fusseder, E Bourne, Alessandro Sette and Bjoern Peters Software ElliPro: a new structur-based tool for the prediction of antibody epitopes. BMC Bioinformatics. 9, 5141-5148 (2008)

29. M Shirai, CD Pendleton, J Ahlers, T Takeshita, M Newman, JA Berzofsky, Helper-cytotoxic $\mathrm{T}$ lymphocyte $(\mathrm{CTL})$ determinant linkage required for priming of anti-HIV CD8+ CTL in vivo with peptide vaccine constructs. J Immunol. 152, 549-556 (1994)

30. K Bharati, R Rani, S Vrati, Evaluation of Japanese encephalitis virus DNA vaccine candidates in rhesus monkeys [Macaca mulatta]. Vaccine. 27, 10-16 (2009). doi:10.1016/j.vaccine.2008.10.050

31. J Kimura-Kuroda, K Yasui, Protection of mice against Japanese encephalitis virus by passive administration with monoclonal antibodes. J Immunol. 141, 3606-3610 (1998)

32. K Murali-Krishna, V Ravi, R Manjunath, Protection of adult but not newborn mice against lethal intracerebral challenge with Japanese encephalitic virus by adoptively transferred virus-specific cytotoxic T lymphocytes: requirement for L3T4+ T cells. J Gen Virol. 77(Pt 4):705-714 (1996)

doi:10.1186/1743-422X-8-232

Cite this article as: Wang et al: Design and evaluation of a multiepitope assembly Peptide (MEAP) against herpes simplex virus type 2 infection in BALB/c mice. Virology Journal 2011 8:232.

\section{Submit your next manuscript to BioMed Central and take full advantage of:}

- Convenient online submission

- Thorough peer review

- No space constraints or color figure charges

- Immediate publication on acceptance

- Inclusion in PubMed, CAS, Scopus and Google Scholar

- Research which is freely available for redistribution 